\title{
An Improved Model for Rosen Type Piezoelectric Transformer for power converter
}

\author{
S.W. Fung \\ Power Electronics Laboratory \\ Department of Electrical \& Electronic \\ Engineering \\ The University of Hong Kong, \\ Pokfulam Road, Hong Kong, China \\ Email: swfung@eee.hku.hk
}

\author{
M.H. Pong \\ Power Electronics Laboratory \\ Department of Electrical \& Electronic \\ Engineering \\ The University of Hong Kong, \\ Pokfulam Road, Hong Kong, China \\ Email: mhp@eee.hku.hk
}

\begin{abstract}
Piezoelectric transformer is attractive because of its small thickness. Rosen Type piezoelectric transformer has high step-up ratio which is advantageous for high voltage power converters. In most cases the load has a range of variable resistances that can greatly affect the output characteristic of a piezoelectric transformer. The conventional model is not sufficient to model the gain characteristics with a wide load range. In this paper a modified model is presented. A simple fictitious resistor is employed to modify the conventional model and it produces excellent match with measurements. Experimental measurements are given to show the merits of the proposed model.
\end{abstract}

\section{INTRODUCTION}

The small thickness of piezoelectric transformer (PT) attracts many researchers to study and develop it for power converters. The Rosen Type piezoelectric transformers are generally considered outstanding which provides high step up ratio and high power density. $[1,2,3]$ A typical application is the backlight power converters of portable computers and hand held devices.

The Piezoelectric transformer has a number of drawbacks as a power conversion component. A major issue is the tricky resonant characteristics that present challenges to provide a suitable control mechanism. In general the operation point should be maintained in the resonant region on the characteristics. The conventional $\mathrm{AC}$ equivalent circuit of Rosen type PT can emulate the relationship between voltage gain and driving frequency but it is insufficient to emulate the relationship between voltage gain and a wide load range. The result generated from the conventional equivalent circuit is different from the practical results on the voltage gain versus load resistance. A correct modeling of voltage gain versus load resistance is needed for PT converter design.

In this study we develop an improved $\mathrm{AC}$ equivalent circuit of Rosen type PT that emulates the voltage gain under a wide load range. The new equivalent circuit is modified from the conventional equivalent circuit. A virtual resistor is developed base on small signal experimental result. This virtual resistor is designed to connect in series with the actual load resistor. Hence, parameters in this new model can be obtained easily through measurement and calculation. More importantly, it is most suitable for computer simulation.

\section{CONVENTIONAL PIEZOELECTRIC TRANSFORMER MODEL}

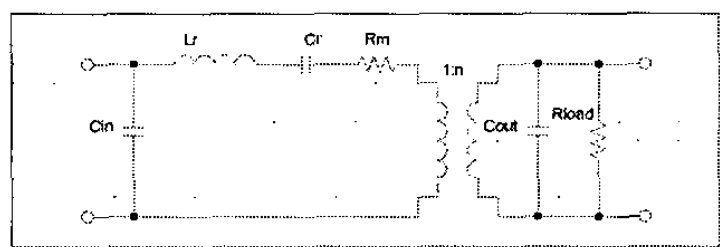

Fig. 1. Conventional equivalent circuit of a piezoelectric transformer

Figure 1 shows a widely accepted piezoelectric transformer model. $[1,4,6]$ Its operation is based on mechanical resonance of the structure whereby electrical energy is produced through exchange of mechanical energy. It is modeled by a resonant circuit.

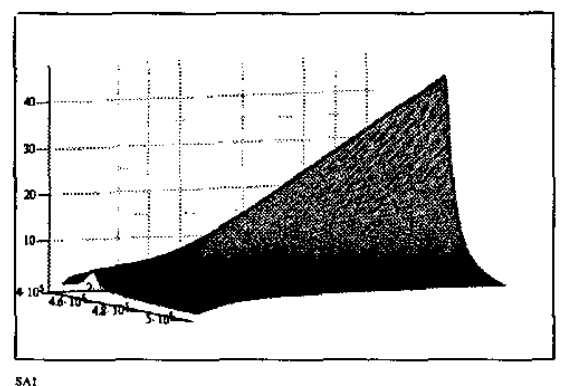

Fig. 2. Voltage gain versus load resistor of conventional PT 
(4WRosen type PT) Vgain vs Loading Resistance

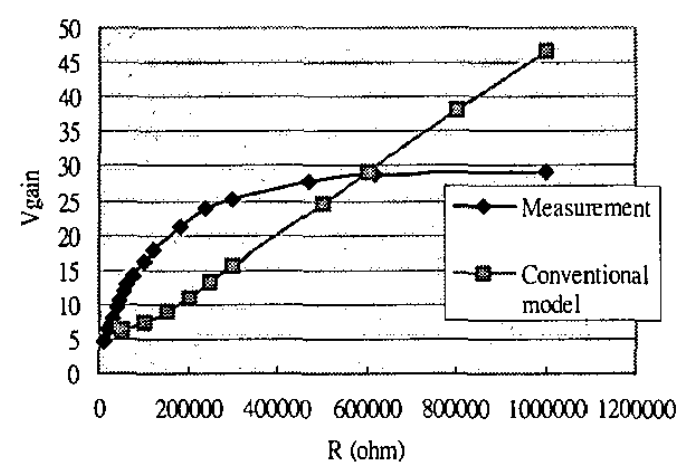

Fig. 3. Voltage gain versus loading resistance $\rightarrow$ Measurement of a practical Rosen type PT and the result from the conventional PT model

The conventional model of a PT consists of a series resonance branch $R_{m} L_{r} C_{r}$, an ideal transformer with step up ratio $n$, an input capacitor $C_{\text {in }}$ and a output capacitor $C_{\text {out }}$ (Figure 1). And the $\mathrm{R}_{\mathrm{L}}$ is the loading resistor.

Figure 2 shows the simulated result of the conventional PT model. It gives the resonant peak in the frequency range concerned. The peak value of the voltage gain rises almost linearly with the load resistance.

The relationship between voltage output, frequency and load resistance is:

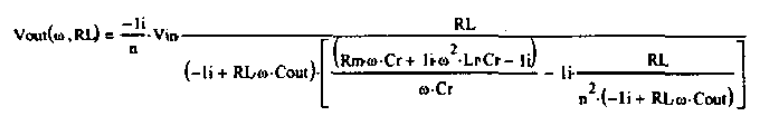

The conventional model of PT (Figure 1) is erroneous on the output voltage versus load resistance characteristics. This is evident by a comparison of the simulated and the experimental result shown in Fig. 3. The simulated result shows a steady increasing voltage with load resistance while the measurements show the voltage gain flattens out. It is because the conventional model is constructed to emulate the resonant frequency at a certain point only. Since a Rosen type PT is usually applied to a range of load resistances, the output voltage variation with load resistance needs some modifications. As shown on Figure 3, the result from conventional model is erroneous in a wide range compared with measurement result from an impedance analyzer HP4194.

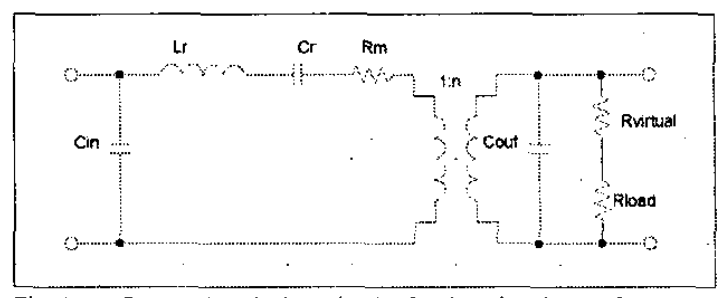

Fig. 4. Proposed equivalent circuit of a piezoelectric transformer

The main interest for Rosen Type piezoelectric transformer is high voltage inverter for cold cathode fluorescent lamp (CCFL). Generally, the piezoelectric transformer is shown to be advantages in current source load application. The instantaneous output voltage follows Ohm's law such that $\mathrm{V}=\left[\times \mathrm{R}_{\text {load }}\right.$. In this manner, having the relations between two variables one can know the characteristic of a piezoelectric transformer in the electrical view. In this research, the relation between output voltage and load resistance is the focus. There are two reasons; output voltage can be obtained easily and is the most reliable measurement data in the practical high voltage application of Rosen Type PT. Secondly, voltage gain $\left(\mathrm{V}_{\text {output }} / \mathrm{V}_{\text {input }}\right)$ is an important indicator of the performance of a Rosen Type piezoelectric transformer.

In order to develop a model that can meet the goal to show the realistic performance of PT, we divide the modeling process into three different stages. These stages are described as follow:

\section{a) Stage I -- Observation of Piezoelectric Transformer}

i. Study the conventional equivalent circuit of piezoelectric transformer by calculation, circuit simulation and graph plotting. Figure 2 shows the calculated result of the conventional piezoelectric transformer equivalent circuit. It shows the instantaneous output voltage gain is nearly directly proportional to the load resistance.

ii. Measure the actual frequency response and voltage gain of a piezoelectric transformer. Also include the environmental factors that affect the electric characteristic of the piezoelectric transformer, such as temperature and mounting method.

Compare the data of conventional model and the practical measurement. Data comparison and analysis develop the direction and method for the Model Setup stage.

b) Stage II - Model Setup

\section{MODEL DEVELOPMENT}


i. The aim of the improved model is to enhance accuracy on reflecting the actual voltage gain versus load resistance.

ii. In Figure 4 we propose a new equivalent circuit model to analyze the voltage gain characteristics of a Rosen piezoelectric transformer. It is based on the conventional piezoelectric transformer equivalent circuit but this model emphasizes on voltage gain performance with different load resistance. In order to simplify the model and calculation procedures, it has an additional virtual resistor $R_{\text {virtual }}$. There is no need to change the resonance components, which are the same as the conventional model.

iii. The parameters of the conventional equivalent circuit can be used directly to construct this new model. This has the advantages that the parameters can be easily generated or even extracted directly from the datasheet. This proposed model is based on practical experiments and observation, the following assumptions are made:

a. the loading is resistive;

b. only small signal is considered.

iv. Tests and observations show that the output characteristics of Rosen Type piezoelectric transformer fits well into the following equation:

$$
\mathrm{G} 2(\mathrm{x} 2)=\frac{\mathrm{x} 2}{\mathrm{R} \lambda+\mathrm{x} 2} \cdot \sigma
$$

This equation is treated as a general equation of our model that describes the practical output characteristic. This general equation will be used to generate some useful parameters and it will be described in detail in the Model Application Section.

v. Calculation and simulation, show that the output curve of conventional piezoelectric transformer model fits well into the following equation:

$$
G l(x l)=\frac{(\beta-\alpha) \cdot(x 1-R \alpha)}{R \beta-R \alpha}+\alpha
$$

The two equations (2)\&(3) together produce the relationship between measurement result and calculation result from a Rosen Type piezoelectric transformer. $R \alpha$ is a value calculated from the upper boundary of the interested load range. $R \beta$ is the value calculated from the lower boundary of the interested load range. Parameters $\alpha$ and $\beta$ are the corresponding gains.
Write the general equation (2) in the form:

$$
G X(R L)=\frac{R L}{R \lambda+R L} \cdot \sigma
$$

Then solve equation (4) by equation (3) and determine the function $R M(R L)$ :

$$
R M(R L)=\frac{A+B+C}{(R \lambda+R L) \cdot(-\beta+\alpha)}
$$

where

$$
\begin{aligned}
& A=R L \cdot R \beta(-\sigma+\alpha) \\
& B=\operatorname{RL} R \alpha(\sigma-\beta) \\
& C=R \lambda(\alpha \cdot \operatorname{R} \beta-\beta \cdot \operatorname{R} \alpha)
\end{aligned}
$$

$R M$ is a function of the actual load resistance RL. $R M$ is a fictitious resistor that modifies the actual load resistance to a modified value. This resistor RM can directly replace the load resistance in the conventional model and produce a modified relationship between load resistance and voltage gain. In order to facilitate computer simulation a virtual resistance $R V$ is extracted to connect in series with the actual load resistor RL. RV(RL) is represented by:

$$
R V(R L)=\frac{A+B+C}{(R \lambda+R L) \cdot(-\beta+\alpha)}-R I
$$

In this stage, the virtual resistance $R V$ is defined and the detail application is described in the following section.

\section{MODEL APPLICATION}

In order to show the application of the new model, we divide the process into 3 steps:

Step l:

Here we want to establish parameters for the equations from practical measurements.

$\mathrm{R} \lambda$ is the parameter to be determined. In order to determine the parameter a resistance value $\times 2$ is picked up from gain measurement with different load resistances. The point $\mathrm{x} 2$ is better chosen from around the desired operating region. The corresponding gain G2 can also be obtained. The 
maximum open circuit gain $\sigma$ can be obtained by measurement or from datasheet. It should be noted that the open circuit gain should not be calculate from the conventional piezoelectric transformer equivalent circuit. This error is shown on figure 3. Parameter $\mathrm{R} \lambda$ can be calculated by solving equation (2). This parameter is largely constant for different choices of $\mathrm{X} 2$.

\section{Step 2:}

Move to the equation (3), parameter $\alpha$ is the voltage gain with respect to a load resistance $R \alpha$ which takes the upper boundary of the interested load range. This is different from $\mathrm{R} \alpha, \beta$ and $\mathrm{R} \beta$ which are taken at the lower boundary of the interested load range.

For example:

If we are interested in the load range $50 \mathrm{k}$ ohm to $300 \mathrm{k}$ ohm,

$\mathrm{R} \beta=50000, \quad \mathrm{R} \alpha=300000$,

then take these two resistances from measurement and get their corresponding voltage gains, $\beta$ and $\alpha$.

Step 3:

Rewrite equation (2) in the form of (4) and put all the defined parameters in function $R M(R L)$. Hence virtual resistance $R V$ can be defined by equation (6). The virtual resistor is a function of the actual load resistance $R_{L}=R_{\text {load }}$.

\section{EXPERIMENTAL VERIFICATION}

The proposed model is evaluated using the gain analysis function of the impedance analyzer HP4194. Two pieces of single layer Rosen type piezoelectric transformers are used, one has maximum 2 watt output and the other one has 4 watt. The PT output terminals are connected to different resistors for testing.

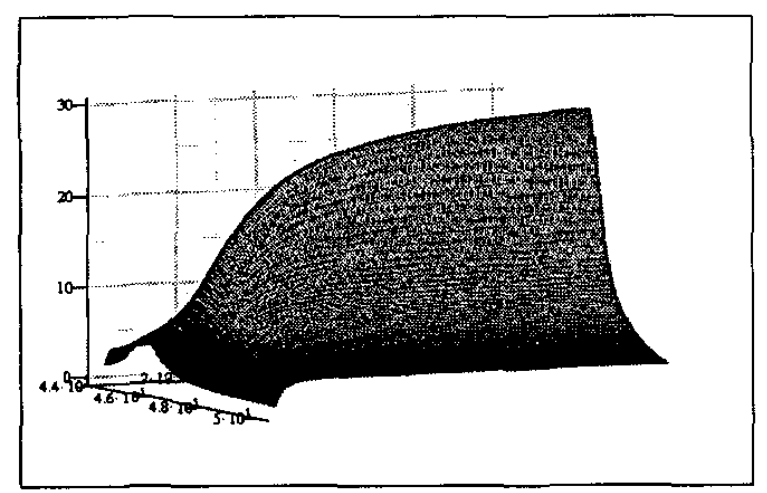

SAl (a)

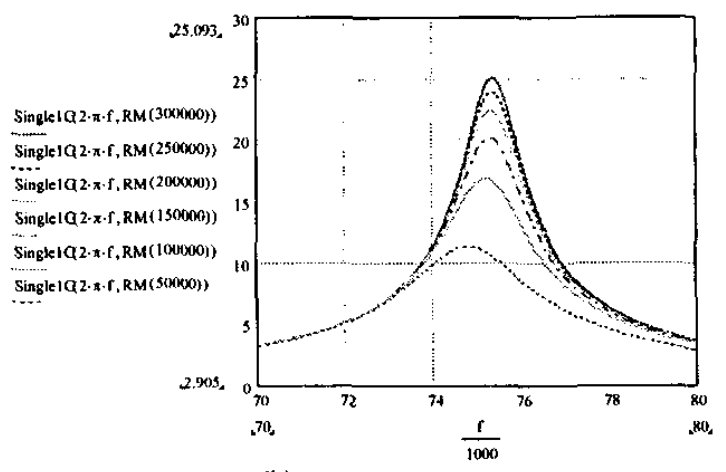

(b)

Fig. 5. MathCad calculation result from proposed PT model; (a)-3D plot Vout/Vin vs loading resistance (RL) and frequency;

(b)-2D plot Vout/Vin vs frequency

Figure 5 presents the experimental result of the 4watt piezoelectric transformer. It shows the calculated results that use the proposed model. (a) \& (b) show the output characteristic is significantly more accurate than the result from conventional model in Figure 2. Figure 6 illustrates the calculated results match closely with the measurement results. Another 2-watt Rosen type PT has been tested and the results are equally accurate.

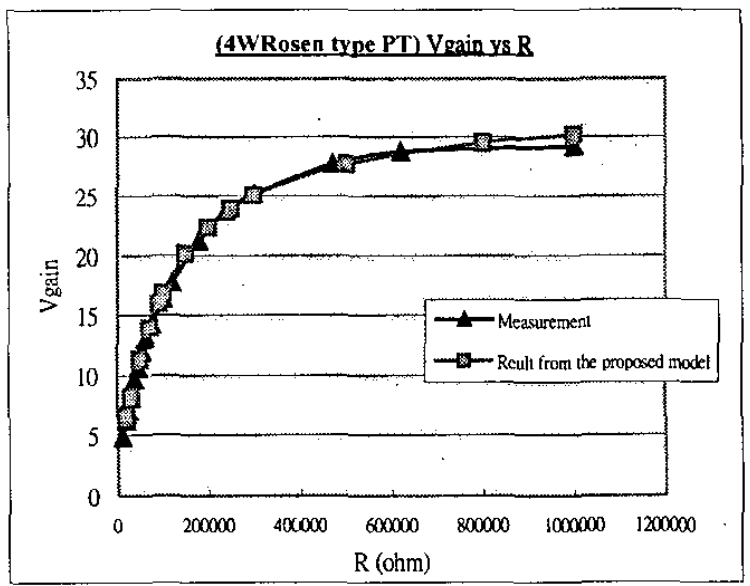

Fig. 6. Comparison between measurement and calculation result from this proposed model. 


\section{CONCLUSION}

Rosen type piezoelectric transformer is an attractive component for portable high voltage applications because of its small thickness and low cost. The conventional piezoelectric transformer model is not sufficient to emulate the load behavior of a Rosen type piezoelectric transformer in a wide load range. In this paper, a modified piezoelectric transformer model is developed to represent the characteristics of voltage gain versus load resistance. Verification results are presented and illustrate the accuracy of this model.

\section{REFERENCES}

[1] Chih-yi Lin, "Design and Analysis of Piezoelectric Transformer Converters" Ph.D Dissertation, Electrical engineering Dept., Virginia Polytechnic Institute and State University, 1997

[2] S. Kawashima, O. Ohnishi et al, "Third Order Longitudinal Mode Piezoelectric Ceramic Transformer and its applicastion to HighVoltage Power Inverter,", NEC Corporation and Yamagata University.

[3] Moo, C.S.; Chen, W.M.; Hsieh, H.K, "Electronic ballast with piezoelectric transformer for cold cathode fluorescent lamps"

[4] Gregory Ivensky, Moshe Shvartsas, and Sam Ben-Yaakov, "Analysis and Modeling of a Piezoelectric Transformer in High Output Voltage Applications.", Ben-Gurion University of the Negev.

[5] M. J. Prieto, J. Diaz, J. A. Martin, F. Nuno, "A Very simple DC/DC Converter Using Piezoelectric Transformer", Universidad de Oviedo - Area de Tecnologia Electronica, Spain, 2000.

[6] M.Sanz, P.Alou, R.Prieto, J.A.Cobos and J.Uceda, "Comparison of different alternatives to drive Piezoelectric Transformers",

Universidad Politecnica de Madrid, ESPIRIT project $\$ 25644,2002$ 